\title{
Epilepsiai
}

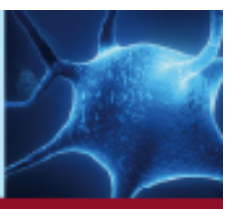

\section{Toward evidence-based severity assessment in rat models with repeated seizures: III. Electrical post-status epilepticus model}

\begin{tabular}{|c|c|}
\hline Journal: & Epilepsia \\
\hline Manuscript ID & EPI-00149-2019 \\
\hline Manuscript Type: & Full length original research paper \\
\hline $\begin{array}{r}\text { Date Submitted by the } \\
\text { Author: }\end{array}$ & 20-Feb-2019 \\
\hline Complete List of Authors: & $\begin{array}{l}\text { Seiffert, Isabel; Ludwig-Maximilians-University (LMU), Institute of } \\
\text { Pharmacology, Toxicology \& Pharmacy } \\
\text { van Dijk, R. Maarten ; Ludwig-Maximilians-University (LMU), Institute of } \\
\text { Pharmacology, Toxicology \& Pharmacy } \\
\text { Koska, Ines; Ludwig-Maximilians-University (LMU), Institute of } \\
\text { Pharmacology, Toxicology \& Pharmacy } \\
\text { Di Liberto, Valentina; University of Palermo, Experimental Biomedicine } \\
\text { and Clinical Neuroscience } \\
\text { Möller, Christina; Ludwig-Maximilians-University (LMU), Institute of } \\
\text { Pharmacology, Toxicology and Pharmacy } \\
\text { Palme, Rupert; University of Veterinary Medicine, Vienna, Department of } \\
\text { Biomedical Sciences } \\
\text { Hellweg, Rainer; Charité, Berlin, Department of Psychiatry and } \\
\text { Psychotherapy } \\
\text { Potschka, Heidrun; Ludwig-Maximilians-University (LMU), Institute of } \\
\text { Pharmacology, Toxicology \& Pharmacy }\end{array}$ \\
\hline Key Words: & Behavior, Stress, Rodent, Basolateral Amygdala, 3R \\
\hline
\end{tabular}

\section{SCHOLARONE




\section{Toward evidence-based severity assessment in rat models with repeated seizures:}

\section{Electrical post-status epilepticus model}

Isabel Seiffert $^{1 *}$, R. Maarten van Dijk ${ }^{1 *}$, Ines Koska ${ }^{1}$, Valentina Di Liberto ${ }^{1,2}$, Christina Möller ${ }^{1}$, Rupert Palme $^{3}$, Rainer Hellweg ${ }^{4}$, Heidrun Potschka ${ }^{1 \#}$

1 Institute of Pharmacology, Toxicology, and Pharmacy, Ludwig Maximilian University of Munich, Munich, Germany.

2 Department of Experimental Biomedicine and Clinical Neurosciences, University of Palermo, Palermo, Italy.

3 Department of Biomedical Sciences, University of Veterinary Medicine, Vienna, Austria.

4 Department of Psychiatry and Psychotherapy, Charité, Berlin, Germany.

* Shared first authorship

\footnotetext{
\# Corresponding author: Prof. Dr. Heidrun Potschka, Königinstr. 16, D-80539 München; telephone number: +49 (0)89 2180-3873; fax number: +49 (0)89 2180-16556; potschka@pharmtox.vetmed.uni-muenchen.de
}

Number of text pages: 23

Number of words: 3983

Number of references: 33

Number of figures: 6

Number of tables: 0 


\section{Abstract \\ Objective:}

Ethical approval of experiments in chronic epilepsy models requires a careful balancing of the expected gain-in-knowledge with the level of distress. Thus, recommendations for evidencebased severity assessment and for classification are urgently needed to the field of preclinical epilepsy research.

Methods:

Therefore, we have completed a comprehensive analysis of alterations in behavioral, biochemical, and physiological parameters in a rat electrical post-status epilepticus model. Selected parameters were repeatedly analyzed during different experimental phases to obtain information about the level of distress throughout the course of the model.

Results:

Behavioral patterns comprised an increase in activity along with a reduction in risk assessment behavior, active social interaction, saccharin preference as well as non-essential, but evolutionary-determined behavior such as nest building and burrowing. Among the biochemical parameters fecal corticosterone metabolites proved to be increased in different phases of the experiment. In the early post-insult phase this increase was reflected by elevated serum corticosterone concentrations. Telemetric recordings demonstrated increases in the level of home cage activity and heart rate in selected experimental phases, but argued against relevant changes in heart rate variability. Comparison between groups with tethered or telemetric recordings including a principal component analysis did not confirm a robust and consistent difference.

Significance:

The present findings further confirm that burrowing behavior and saccharin preference might serve as valid parameters for severity assessment in chronic epilepsy models. Considering the course of alterations providing evidence for a more pronounced level of distress in the early 
phase following status epilepticus (SE), we suggest a classification of the electrical post-SE model as severe. This suggestion may serve as a guidance for laboratory-specific evaluations. Unexpectedly, comparison between data from animals with tethered and telemetric recording did not support the validity of telemetry as a putative refinement measure in this rat post-SE model.

Keywords: Behavior, Stress, Rodent, Basolateral Amygdala, 3R

\section{Key Point Box:}

- The data indicate that the assessment of burrowing behavior and saccharin preference can serve as indicators of severity.

- The findings suggest classification of the electrical post-SE model as severe.

- No clear indication was found for a decrease of severity due to telemetric recordings over tethered recordings in the model used. 


\section{Introduction}

Post-SE models constitute important paradigms allowing to study the mechanisms of epilepsy development and to identify and evaluate biomarkers and target candidates for anticonvulsant, anti-epileptogenic, and disease-modifying approaches. ${ }^{1}$ Considering the complex cellular and network alterations characterizing epilepsy development and manifestation ${ }^{2}$ it is still impossible to completely replace in-vivo experiments by in-vitro studies in this field of research. It is therefore all the more important to consistently apply the other two concepts of the $3 \mathrm{R}$ principle, i.e. reduction and refinement. ${ }^{3-5}$ Refinement comprises the selection of the least burdensome model, which allows addressing the research hypothesis with a good scientific quality and informative data value. ${ }^{6}$ Moreover, it is based on the application of measures that minimize the severity of the experimental procedures. Lidster and colleagues (2016) have intensely discussed the necessity to assess specific refinement measures for rodent epilepsy models and have pointed out that a gain in knowledge in evidence-based severity assessment is urgently needed 2.

As described previously ${ }^{7}$, the development and application of comprehensive severity assessment schemes will provide an improved basis for evidence-based severity classification allowing to carefully assess differences between models and the impact of putative refinement measures. Recently, we have provided first scientific evidence for the classification of the kindling paradigm along with a recommendation for the application of selected parameters for a laboratory-specific evaluation.

In chronic models with spontaneous recurrent seizures, animals experience different experimental influences during various phases from induction of the brain insult to the latency phase to the chronic phase with epilepsy manifestation. ${ }^{1}$ Most of the research questions require a thorough video/EEG monitoring to capture information about the frequency, severity, and duration of spontaneous seizures. Monitoring is thereby based on tethered recording with or without swivel systems or on telemetric recordings. Whereas the first method can result in 
mobility restrictions with tractive forces by the cable, telemetric recordings require additional subcutaneous or abdominal implantation of a transmitter. ${ }^{2 ;} 8 ; 9$ It has been hypothesized by Lidster et al. ${ }^{2}$ that telemetric recordings may reduce the burden and stress for the animals during monitoring phases. However, respective scientific proof is still pending.

Electrical post-SE models have been developed as an alternate for the application of chemoconvulsants including pilocarpine or kainic acid avoiding potential influences of the proconvulsant compounds with test compounds. ${ }^{1 ; 10}$ Moreover, there has been the impression that electrical models may cause a milder phenotype associated with less severe neuropathological alterations thereby resulting in a model that may be closer to the clinical scenario in human patients. $1 ; 11$

In this study, we have assessed the impact of an electrical post-SE model on a comprehensive set of behavioral, biochemical and physiological parameters, which can reflect distress and an impact on the well-being of the animals. The model has been developed with several modifications ${ }^{11}$ based on an earlier approach by McIntyre et al. ${ }^{12}$. It is based on 25-min stimulation of the basolateral amygdala resulting in the induction of a self-sustained SE. It has been selected as a representative example of an electrical post-SE model for the current study. This study is a part of a series about the severity classification of three commonly used rat epilepsy models (I. Kindling model published by Möller et al ${ }^{7 ; 13}$, II. Chemical post-SE model submitted in parallel by Koska et al. and the present study; III Electrical post-SE model.) 


\section{Material and Methods}

\subsection{Animals}

In total 63 female Sprague-Dawley rats (200-224 g, Envigo, the Netherlands) were used for tethered and telemetry recording. Additional information concerning the housing of the animals can be found in supplementary material S1. Animals were randomly divided in subgroups (www.randomizer.org). Out of the 63 animals 44 were used for tethered recordings (12 naive, 14 sham and $18 \mathrm{SE}$ ) and 19 for the telemetric recordings (6 sham and $13 \mathrm{SE})$. Examined time points in this project (see Figure S1 and S2 for a timeline) are the recovery phase post-surgery and three post-SE time points: the first week post-SE, "post-insult phase", the $2^{\text {nd }}$ to $7^{\text {th }}$ week post-SE "latency phase" and from the $8^{\text {th }}$ week on to the end of study "chronic phase". Throughout the text animals with history of SE are labelled as "post-SE" in the early and latency phase. In the chronic phase they are labelled as "epilepsy". In total 18 animals (12 in the tethered and 6 in the telemetry group) out of the initial 63 animals were excluded for various reasons. Six animals died as a result of the SE induction, four animals lost their electrode, and four animals were scarified due to other health concerns such as tumours or wound inflammation. Four animals (two in the tethered and two in the telemetry group) did not develop spontaneous recurrent seizures; these animals were excluded in the group wise comparisons but included in the correlation matrix and principal component analysis.

The study was approved by the Committees of the Government of Upper Bavaria (reference number 55.2-1-54-2532-105-16, 55.2-1-54-2532-011-2015) and was conducted in accordance with the German Animal Welfare act and the EU directive 2010/63/EU. All procedures followed the ARRIVE guidelines and the Basel declaration including the 3R concept.

\subsection{Electrode and telemetry implantation, electrical stimulation}


The electrode implantation and the electrical induced SE was performed according to Walker et al. ${ }^{14}$ to the right basolateral amygdala (BLA) (AP $\left.-2.2, \mathrm{~L}+4.7, \mathrm{~V}+8.5 \mathrm{~mm}\right)$. The implantation of the telemetry device was done as described by Möller et al. ${ }^{13}$. For a detailed description of the implantation of the transmitter and the electrical stimulation see supplementary material S1.

\subsection{Tethered and telemetric recordings}

In order to detect spontaneous recurrent seizures all stimulated animals were video and EEG monitored for 14 days eight weeks post-SE. The tethered group was monitored as described by Walker et al. ${ }^{14}$. The EEG, ECG and activity of the telemetry-implanted Sham and SE animals were in addition recorded for 48 hours during baseline and the three post-SE phases. A description of the telemetric monitoring and analysis of time domain and frequency domain can be found in supplementary material S1.

\subsection{Evaluation of behavioral and biochemical parameters}

Evaluation of all behavioral and biochemical parameters were performed as previously described by Möller et al. ${ }^{7}$.A detailed timeline can be found in supplementary figure 1 and 2 . Details concerning the statistical approach can be found in supplementary material S1.

\section{Results}

\subsection{Induction of status epilepticus and development of spontaneous recurrent seizures}

Twenty-five (tethered: 14, telemetry: 11) out of 29 stimulated rats developed a type $3 \mathrm{SE}$ according to Brandt et al. ${ }^{11}$ while four animals from the tethered group generated a type 2 SE. 
Twenty-one stimulated rats (tethered: 12; telemetry: 9) reached the chronic phase. Fifteen rats exhibited seizures during the EEG monitoring (Figure S.3; tethered: $n=8$, telemetry: $n=6$ ) with a mean seizure duration of $623 \mathrm{~s}(\mathrm{SD}=718$, median $=291)$ in the tethered and $581 \mathrm{~s}(\mathrm{SD}=$ 722 , median $=283)$ in the telemetry group. The tethered group had a mean seizure frequency of $13(\mathrm{SD}=18.7$, median $=6)$ and the telemetry group of $9.6(\mathrm{SD}=12.5$, median $=5)$ in 14 days.

\subsection{Impact on nest building and soiling}

In animals with SE a reduction of nest complexity became only evident one week following SE (Figure 1A,B). No alterations were observed at later time points during epileptogenesis (Figure S4). Following SE, the level of soiling (Figure S5) reached higher levels during the latency phase in animals prepared for tethered recordings (Figure S5D). A decreased distribution observed in the early phase following SE in animals with telemetry transmitters (Figure S5C).

\subsection{Impact on the Grimace scale, behavior in the burrowing paradigm and the open field}

The Grimace scale reached scores between 0.8 and 0.9 on the first post-surgical day. Scores gradually decreased reaching baseline levels again between the $2^{\text {nd }}$ and $3^{\text {rd }}$ post-surgical day (Figure S6A,B). Surgery did not result in any significant weight loss (Figure S6C,D). In contrast, significant weight loss occurred following SE. This was regained over the following two weeks (Figure S6E,F). From the third week till the end of the projects the stimulated animals exhibited an increased body weight as compared to both control groups.

In the open field paradigm measured in the chronic phase, electrode-implanted rats without SE spent more time in the outer ring (Figure 1C) and less time in the center of the open field (Figure S7A). Animals with epilepsy and previous tethered recordings exhibited an increased distance moved (Figure 1E) and a reduced frequency of immobility phases (Figure S7E). In contrast, 
animals with epilepsy and telemetric recordings showed reduced thigmotaxis and increased time in the middle ring (Figure 1D,H).

One week after surgery, electrode-implanted animals showed decreased burrowing behavior compared to naive animals (Figure 2A). Following SE, animals exhibited a prolonged latency to initiate burrowing behavior and a reduced amount of gravel burrowed (Figure 2C,D). The impact proved to be evident throughout all phases of epileptogenesis regardless of the EEG monitoring approach, i.e. telemetric (Figure S8) or tethered (Figure 2E-H).

\subsection{Impact on social interaction, anxiety-associated and anhedonia-associated behavior}

In animals with epilepsy manifestation, the time spent with active social interaction proved to be significantly reduced (Figure 3A,B).

Animals with tethered recordings did not exhibit behavioral alterations in the BWB paradigm (Figure 3C,E; S9E,G). In contrast, we observed a lower number of stretching postures and entries in the white box in animals with telemetric recordings (Figure 3D,F).

In the EPM, an increased level of activity was evident in animals with epilepsy and telemetric recordings (Figure S9B,D). In contrast, activity proved to be in the control range in animals with epilepsy and tethered recordings. Both groups spent less time in the center of the maze as compared to their respective control groups (Figure 3G,H).

Anhedonia-associated behavior was assessed based on saccharin consumption. Regardless of the preceding EEG monitoring approach, animals with epileptic seizures showed a reduced preference of saccharin in comparison with the control groups (Figure 3I,J).

\subsection{Impact on biochemical parameters}

Neither adrenal gland weight nor any of the serum and hair biochemical parameters were affected in the chronic phase (Figure S10A-F; S11). 
As we wanted to avoid invasive sampling during the experiment, analysis during earlier experimental phases has been restricted to feces samples (Figure 3K,L). Two days following SE, we demonstrated increased concentration of feces corticosterone metabolites in animals prepared for tethered recordings and a trend for a respective increase in animals prepared for telemetric recordings. Moreover, elevated fecal corticosterone metabolite levels were evident before and following telemetric monitoring in the respective group of rats.

Considering the early increase of fecal corticosterone metabolites following SE, we were interested to additionally analyze serum corticosterone during other experimental phases. Serum samples were available from rats from a different study, in which animals with an electrode implantation only were sacrificed two and ten days following SE (Figure S12). Analysis of their serum samples revealed increased corticosterone levels two days following SE.

\subsection{Impact on home cage activity and heart rate}

Home cage activity (Figure 4A,B) assessed by telemetric recordings during the light phase (=resting phase) proved to be increased in the early post-insult phase when compared to baseline measurement. Following epilepsy manifestation, analysis of recordings during the dark phase (=activity phase) demonstrated activity levels exceeding those during baseline recordings in the latency phase.

In the early phase following SE, comparison with respective baseline data revealed an increase in heart rate associated with a decrease in the $\mathrm{NN}$ interval during the resting phase of the animals (Figure 4C,D; S13A,B). Moreover, in the active phase the NN interval proved to be decreased four weeks following SE. Group differences between implanted rats and rats with SE were only observed during the early post-insult (dark phase) and the latency phase (light and dark phase). Aiming to obtain information about the total variability of the heart rate, we analysed the standard deviation of $\mathrm{NN}$ intervals $(=\mathrm{SDNN})$. In both groups SDNN remained in the range of 
baseline measurements throughout the experiment (Figure S14). Differences between groups were evident during selected experiment phases. However, it needs to be considered that a group difference was already evident during the baseline dark phase recordings.

Information about short-term variability and spontaneous adjustment of heart rate was obtained by analyses of further parameters (RMSSD = root mean square of successive differences; NN9= percent of subsequent $\mathrm{NN}$ intervals, which deviate more than $9 \mathrm{~ms}$; $\mathrm{pNN} 9=$ proportion derived by dividing NN9 by the total number of $\mathrm{NN}$ intervals). None of these parameters proved to be affected by the experimental procedures (data not shown).

An additional analysis of the frequency-domain did not reveal relevant experiment-associated alterations in comparison with baseline measurements (Figure S15). A group difference was only evident in the latency phase with animals following SE exhibiting a lower low-frequency band to high-frequency band ratio ( $\mathrm{LF} / \mathrm{HF}$ with $\mathrm{LF}=0.1-1.0 \mathrm{~Hz}$ and $\mathrm{HF}=1.0-3.5 \mathrm{~Hz})$.

\subsection{Correlation matrix of all measured variables}

Two correlation matrices were created, one comparing all variables recorded in animals with a tethered recording (Figure 5) and one with al variables recorded in animals with a telemetric recording (Figure S16). As the number of significant correlations are too numerous to be listed here only the selected noteworthy findings will be mentioned.

Several groups of variables stand out for sharing a high number of correlations with other variables. Total weight gain and food intake measured at various time points are highly correlated with each other and in turn both correlate with a number of other measures such as the latency to start burrowing, social interaction and both the Irwin and Grimace scores. Likewise, both social interaction and burrowing behavior correlate with each other and both show strong correlations with the different scoring schemes.

The correlation matrix within the group of animals which underwent telemetric recordings shares many similarities with the correlation matrix of the animals which underwent tethered 
recordings. When focusing on the correlations with the heart rate and heart variability variables several notable observations can be made. First of all, the heart rate and heart rate variability variables recorded four weeks post-insult show significant correlations with the majority of behavioral and biochemical measures. Secondly, many of the behavioral variables measured at 11-12 weeks post-insult show stronger correlations with heart rate variables measured during four weeks post-insult as opposed to the time point closer to the insult, one week post-insult or closer to the behavioral recordings, nine weeks post-insult.

\subsection{Principal component analysis}

Using the combined data from the stimulated animals from both the tethered as well as the telemetrically recorded groups a principal component analysis (PCA) was performed using only those variables which were measured following the monitoring period, allowing the investigation of any possible effect on behavior due to a difference in tethered vs telemetric recording (Figure 6). The first two principal components $(\mathrm{PC} 1,2)$ represent a total of $41.44 \%$ of the variance (PC1: $24.80 \%, \mathrm{PC} 2: 16.63 \%)$. The two groups are diagonally separated along both PC1 $(\mathrm{F}(3,17)=3.91, \mathrm{p}=0.027)$ and $\mathrm{PC} 2 \quad(\mathrm{~F}(3,17)=3.366, \mathrm{p}=0.043)$. The behavioral paradigm which has a prominent impact on both PC1 and PC2 separating the two groups is the EPM, which occupies the top four parameters along PC2; number of head dips, time spent in the open arms (in total and in the outer 1/3) and on the opposite site the time spent in the closed arms, while also contributing to PC1; again time spent in the closed arms and in the outer $1 / 3$ of the open arms. The second most frequent paradigm is the open field test, which also features on both PC1 and PC2 with the total time spent in the center, the number of rearing postures, total distance moved and the time spent immobile.

\section{Discussion}


Whereas electrical post-SE models in rodents constitute an important tool in experimental epileptology research ${ }^{1}$, the impact on experimental animals needs to be carefully considered for ethical evaluation of animal experiment proposals and for efforts to minimize the burden for the develop refinement measures ${ }^{2}$. Therefore, we have assessed behavioral, biochemical, and physiological parameters throughout different experimental phases based on a comprehensive set of parameters.

In most studies the first intervention comprises stereotactic surgery with implantation of a depth electrode, which in electrical models might serve as the stimulation and recording electrode at the same time 11;12; 15-17. Following implantation of an electrode in the basolateral amygdala, we only observed minor acute and long-term behavioral alterations with a reduced amount of gravel burrowed one week following surgery and a decreased time in the center of the open field 18 weeks following surgery. Along with Grimace scale analysis during the first week following surgery, our data suggest a transient phase of compromised wellbeing in the early post-surgical phase. However, later analyses of behavior and biochemical data during the chronic phase did not indicate a chronically increased level of distress as a consequence of the implant.

All parameters that could be repeatedly assessed without invasive procedures and with stable data despite repeated assessment, were analyzed during the early post-insult phase and the latency phase of the post-SE model.

As expected a transiently compromised wellbeing became evident based on reduced nest complexity as well as body weight one week following SE. The body weight of recovered, stimulated animals recuperated reaching a higher body weight starting from the third week postSE to the end of the project. Whereas nest complexity levels normalized in subsequent phases, burrowing behavior proved to be reduced throughout all post-SE phases including the latency phase. These data should be interpreted in the context of previous studies demonstrating that, 
both, nest building and burrowing behavior can be analyzed as indicators of distress and pain associated with experimental procedures in laboratory rodents ${ }^{18-21}$.

As a short- and long-term consequence of prolonged seizure activity, fecal corticosterone metabolite levels proved to be increased in different phases of the study. However, the alterations of this biochemical stress marker were not very robust as effects were often only observed in one of the animal groups prepared for tethered or telemetric recordings and were not always reflected by alterations in serum or hair corticosterone. The difference between animal groups might also be due to a high level of variance and the smaller $\mathrm{n}$ in the telemetryimplanted group.

As we have demonstrated in a separate group of animals, increased serum corticosterone can be observed in the early post-insult phase. For the interpretation of serum corticosterone levels and fecal corticosterone metabolite data it needs to be considered that these might not only reflect stress but also a direct influence of seizure activity on the hypothalamic-pituitary-adrenal $\operatorname{axis}^{22}$.

Taken together the data confirm a more pronounced detrimental impact on wellbeing in the early post-SE phase, and a milder influence during the latency phase. In the chronic phase, the more detailed analysis of behavioral parameters indicated an elevated level of anhedoniaassociated behavior, and reduced behavioral patterns, for which rodents normally exhibit a high level of motivation. When considering the entire experiment, the level of severity proved to be significantly higher as in the amygdala-kindling paradigm with repeated electrical induction of convulsive seizures ${ }^{7}$, and in a comparable range as in the pilocarpine post-SE model (Koska et al., manuscript submitted in parallel) although relevant differences were also observed between the chemical and electrical model. Therefore, we suggest a classification as severe based on the European expert working group report ${ }^{23}$. As already emphasized in our previous publication ${ }^{7}$, the laboratory specific conditions need to be taken into account, so that a suggestion can only provide a basal guidance for ethical committee members and scientists. 
One main aim of our series of studies is to identify and validate parameters that are suitable for severity classification of epilepsy models, and that might be applied for assessment of new models as well as for evidence-based development of refinement measures according to the 3Rprinciple. The present data further confirm burrowing as a more sensitive indicator of compromised wellbeing as compared to nest building. Moreover, we would like to highlight the saccharin preference test as an easy-to-apply test, which is performed in the home cage without any intervention necessary. Respective data are in line with our findings from the pilocarpine post-SE model (Koska et al., manuscript submitted in parallel).

Among the biochemical parameters fecal corticosterone metabolite analysis seems to be more sensitive as compared to corticosterone analysis in serum or hair ${ }^{24}$. However, as discussed above the interpretation requires more complex considerations and caution in epilepsy models. Only relatively limited effects were observed by additional telemetric analysis including an increased resting activity, heart rate, and $\mathrm{NN}$ interval in selected daytime and experimental phases. Alterations proved to be most pronounced in the early post-insult phase suggesting a disturbed resting phase with elevated activity levels and heart rate. Heart rate elevations have previously been discussed as a marker of distress in laboratory rodents exposed to surgical intervention, tumor models, or a sepsis model ${ }^{8 ; 25-28}$.

In this context, it is of interest that heart rate variability has been reported to be differentially affected by chronic stress exposure with a decrease in time-domain indices and an increase in frequency domain indices ${ }^{29}$. The lack of any relevant and robust alterations in the electrical post-SE model may thus argue against a high level of stress.

The results of the burrowing test and saccharin preference tests proved to correlate with a large number of other measures such as food intake, nest building, social interaction, Irwin and Grimace scoring and different measures of heart rate and heart rate variability measures. Taking into account that these tests are easy to apply, these findings question the additional informative 
value of telemetric activity and electrocardiographic analysis considering the tremendous experimental effort and high costs of these analyses.

Finally, we were eager to obtain further information whether replacement of tethered seizure monitoring by telemetric seizure monitoring reduces the burden for the animals as suggested by Lidster et al. $(2016)^{2}$. Our findings from the pilocarpine model revealed that minor differences can be observed between animals with tethered and telemetric recordings with evidence for a higher level of distress in the group with tethered monitoring (Koska et al., manuscript submitted in parallel).

In the present study, the more comprehensive surgical procedure with subcutaneous implantation of a telemetry transmitter did not result in more pronounced behavioral alterations in the early post-surgical phase. These data indicate, that additional implantation of the transmitter might not increase the severity of the surgical intervention in a significant manner. In the chronic phase, behavioral and biochemical alterations were rather comparable, when comparing rats with tethered vs. telemetric monitoring.

In a principal component analysis, the parameters influencing the separation between the tethered and telemetric groups were recorded in the EPM and open field, suggesting a difference in locomotion between the two groups. Interestingly, it was the group with telemetric recordings which showed higher levels of velocity and distance moved as compared to their electrodeimplanted control group. Whether this effect is due to the tethered group being somewhat restraint in their movement during the two weeks prior to behavioral testing, due to a difference in experienced stress or due to a batch difference between the two experimental groups requires more detailed investigation.

In support of our findings from the pilocarpine post-SE model (Koska et al., submitted), the present data further confirm that burrowing behavior and saccharin preference might serve as valid parameters for severity assessment in chronic epilepsy models. 
Considering the course of alterations in behavioral, biochemical, and physiological parameters with a transient more pronounced impact on wellbeing during the early post-SE phase, we suggest a classification as severe as a guidance for laboratory-specific evaluations.

Comparison between data from animals with tethered and telemetric recording did not indicate pronounced differences in the level of distress among these groups. Thus, in view of previous findings from the pilocarpine post-SE model (Koska et al., submitted), the validity of telemetric recordings as a putative refinement measure might differ depending on the epilepsy model. 


\section{Acknowledgements}

The project was supported by grants of Deutsche Forschungsgemeinschaft (FOR 2591, GZ: PO681/9-1). The authors thank Uwe Birett, Sarah Driebusch, Sieglinde Fischlein, Katharina Gabriel, Andreas Kutschka, Edith Klobetz-Rassam, Claudia Siegl, Isabella Waclawcyk, Silvia Saft, Sabine Sass, Regina Rentsch, Katharina Schönhoff and Sabine Vican for their excellent technical assistance and Prof. Dr. Clemens Kirschbaum (Dresden LabService GmbH) for the analysis of the hair samples.

\section{Disclosure}

The authors declare that they have no competing interest.

\section{Ethical Publication Statement}

We confirm that we have read the Journal's position on issues involved in ethical publication and affirm that this report is consistent with those guidelines.

\section{References}

1. Gorter JA, van Vliet EA, Lopes da Silva FH. Which insights have we gained from the kindling and poststatus epilepticus models? J Neurosci Methods 2016;260:96-108.

2. Lidster K, Jefferys JG, Blumcke I, et al. Opportunities for improving animal welfare in rodent models of epilepsy and seizures. J Neurosci Methods 2016;260:2-25.

3. Rusche B. The 3Rs and animal welfare - Conflict or the way forward? ALTEX 2003;20:63-76.

4. Blakemore C, MaCarthur Clark J, Nevalainen T, et al. Implementing the 3Rs in neuroscience research: a reasoned approach. Neuron 2012;75:948-950.

5. Graham ML, Prescott MJ. The multifactorial role of the 3Rs in shifting the harm-benefit analysis in animal models of disease. Eur J Pharmacol 2015;759:19-29.

6. Aske KC, Waugh CA. Expanding the 3R principles: More rigour and transparency in research using animals. EMBO Rep 2017;18:e201744428.

7. Möller C, Wolf F, van Dijk RM, et al. Toward evidence-based severity assessment in rat models with repeated seizures: I. Electrical kindling. Epilepsia 2018;59:765-777.

8. Cesarovic N, Jirkof $P$, Rettich $A$, et al. Implantation of radiotelemetry transmitters yielding data on ECG, heart rate, core body temperature and activity in free-moving laboratory mice. J Vis Exp 2011:e3260.

9. Lundt A, Wormuth C, Siwek ME, et al. EEG Radiotelemetry in Small Laboratory Rodents: A Powerful State-of-the Art Approach in Neuropsychiatric, Neurodegenerative, and Epilepsy Research. Neural Plast 2016;2016:8213878.

10. Löscher W. Animal models of epilepsy for the development of antiepileptogenic and diseasemodifying drugs. A comparison of the pharmacology of kindling and post-status epilepticus models of temporal lobe epilepsy. Epilepsy Res 2002;50:105-123. 
11. Brandt $C$, Glien $M$, Potschka $H$, et al. Epileptogenesis and neuropathology after different types of status epilepticus induced by prolonged electrical stimulation of the basolateral amygdala in rats. Epilepsy Res 2003;55:83-103.

12. McIntyre DC, Nathanson D, Edson N. A new model of partial status epilepticus based on kindling. Brain Res 1982;250:53-63.

13. Möller C, van Dijk RM, Wolf F, et al. Impact of repeated kindled seizures on heart rate rhythms, heart rate variability, and locomotor activity in rats. Epilepsy Behav 2019;92:36-44.

14. Walker A, Russmann V, Deeg CA, et al. Proteomic profiling of epileptogenesis in a rat model: Focus on inflammation. Brain Behav Immun 2016;53:138-158.

15. Nissinen J, Halonen T, Koivisto E, et al. A new model of chronic temporal lobe epilepsy induced by electrical stimulation of the amygdala in rat. Epilepsy Res 2000;38:177-205.

16. Gorter J, Van Vliet E, Aronica E, et al. Progression of spontaneous seizures after status epilepticus is associated with mossy fibre sprouting and extensive bilateral loss of hilar parvalbumin and somatostatin-immunoreactive neurons. Eur J Neurosci 2001;13:657-669.

17. Shirasaka $Y$, Wasterlain CG. Chronic epileptogenicity following focal status epilepticus. Brain Res 1994;655:33-44.

18. Jirkof P. Burrowing and nest building behavior as indicators of well-being in mice. $J$ Neurosci Methods 2014;234:139-146.

19. Rutten K, Gould SA, Bryden L, et al. Standard analgesics reverse burrowing deficits in a rat $\mathrm{CCl}$ model of neuropathic pain, but not in models of type 1 and type 2 diabetes-induced neuropathic pain. Behavioural brain research 2018;350:129-138.

20. Wodarski R, Delaney A, Ultenius $C$, et al. Cross-centre replication of suppressed burrowing behaviour as an ethologically relevant pain outcome measure in the rat: a prospective multicentre study. Pain 2016;157:2350-2365.

21. Hohlbaum K, Bert B, Dietze $S$, et al. Severity classification of repeated isoflurane anesthesia in C57BL/6JRj mice-Assessing the degree of distress. PLoS One 2017;12:e0179588.

22. O'Toole KK, Hooper A, Wakefield S, et al. Seizure-induced disinhibition of the HPA axis increases seizure susceptibility. Epilepsy Res 2014;108:29-43.

23. Union E. Directive 2010/63/EU of the European Parliament and of the Council of 22 September 2010 on the protection of animals used for scientific purposes 2010:33-79.

24. Scorrano F, Carrasco J, Pastor-Ciurana J, et al. Validation of the long-term assessment of hypothalamic-pituitary-adrenal activity in rats using hair corticosterone as a biomarker. FASEB J 2015;29:859-867.

25. Rowan WH, 3rd, Campen MJ, Wichers LB, et al. Heart rate variability in rodents: uses and caveats in toxicological studies. Cardiovasc Toxicol 2007;7:28-51.

26. Arras $M$, Rettich $A$, Cinelli $P$, et al. Assessment of post-laparotomy pain in laboratory mice by telemetric recording of heart rate and heart rate variability. BMC Vet Res 2007;3:16.

27. Rudiger $A$, Jeger $V$, Arrigo $M$, et al. Heart rate elevations during early sepsis predict death in fluidresuscitated rats with fecal peritonitis. Intensive Care Med Exp 2018;6:28.

28. Thireau J, Zhang BL, Poisson D, et al. Heart rate variability in mice: a theoretical and practical guide. Exp Physiol 2008;93:83-94.

29. Park SE, Park D, Song KI, et al. Differential heart rate variability and physiological responses associated with accumulated short- and long-term stress in rodents. Physiol Behav 2017;171:21-31.

Figure legends

Figure 1, Nest building activity and open field locomotor activity. A \& B: Nest building scores are significantly reduced one week post-SE induction in both the tethered and the telemetry 
group $(\mathrm{A}:(\mathrm{F} 2,31)=8.591, \mathrm{P}=0.0048 ; \mathrm{B}: \mathrm{P}=0.0246$, epilepsy against both control groups $\mathrm{P}<0.05)$. C-H: measures of locomotor activity measured in the open field. In the tethered group sham animals spent significantly more time at the wall compared to the naive animals $(\mathrm{C}$ : $\mathrm{F}(2,29)=3.524, \mathrm{P}=0.0427$, sham against naive $\mathrm{P}<0.05)$ while in the telemetry group the sham animals spent significantly more time at the wall compared to the Epilepsy group $(\mathrm{D}, \mathrm{P}=$ 0.0086). Animals with epilepsy move greater distances compared to the sham group in the tethered group $(\mathrm{E}: \mathrm{F}(2,29)=3.791, \mathrm{P}=0.2073$, epilepsy against sham $\mathrm{P}<0.05)$ but not in the telemetry group (F). Lastly, animals with epilepsy spent more time in the middle of the arena compared to the sham group in the telemetry group $(\mathrm{H}: \mathrm{P}=0.0086)$ while no differences were found in the tethered group $(\mathrm{G})$. Error bars indicate standard error of the mean. $*=\mathrm{P}<0.05$ and $* *=P<0.01$. Total $n$ for the tethered group: naive $n=11$, sham $n=11$, Post-SE $n=12$, epilepsy $n=10$. Total $n$ for the telemetry group: sham $n=6$, epilepsy $n=7$.

Figure 2, Burrowing behavior in animals monitored with a tethered connection. A, C, E \& G: amount of burrowed gravel, B, D, F \& H: latency to start burrowing. Animals burrowed significantly less gravel one week post-surgery $(\mathrm{A}: \mathrm{P}=0.0313)$ and following $\mathrm{SE}$ induction during the early phase $(\mathrm{C}: \mathrm{F}(2,30)=18.93, \mathrm{P}<0.0001$, epilepsy against both control groups $\mathrm{P}<0.001)$, latency phase $(\mathrm{E}: \mathrm{F}(2,30)=8.438, \mathrm{P}=0.0002$, epilepsy against both control groups $\mathrm{P}<0.05)$ and the chronic phase $(\mathrm{G}: \mathrm{F}(2,28)=9.477, \mathrm{P}=0.0007$, epilepsy against both control groups $\mathrm{P}<0.01$ ) compared to both naive and sham animals. In addition, animals had a longer latency to start burrowing during the three phases post-SE induction $(\mathrm{D}: \mathrm{F}(2,30)=11.19 \mathrm{P}=$ 0.0002, epilepsy against both control groups $\mathrm{P}<0.01$; F: $\mathrm{F}(2,30)=162.1, \mathrm{P}<0.0001$, epilepsy against both control groups $\mathrm{P}<0.001 \& \mathrm{H}: \mathrm{F}(2,28)=37.44, \mathrm{P}<0.0001$, epilepsy against both control groups $\mathrm{P}<0.001)$. Error bars indicate standard error of the mean. $*=\mathrm{P}<0.05$, ** $=\mathrm{P}<$ 0.01 and $* * *=\mathrm{P}<0.001$. Total $\mathrm{n}$ for the tethered group: naive $\mathrm{n}=10$, sham $\mathrm{n}=11$, Post-SE $\mathrm{n}=12$, epilepsy $\mathrm{n}=10$. 
Figure 3, Social interaction, anxiety-associated, anhedonia-associated behavior and fecal corticosterone metabolite concentration (FCM). A \& B: Time spent in social interaction, animals with epilepsy spent significantly less time in active social interaction compared to control groups in both the tethered $(\mathrm{A}: \mathrm{F}(2,11)=52.32, \mathrm{P}<0.0001$, epilepsy against both control groups $\mathrm{P}<0.001$ ) as well as the telemetry group ( $\mathrm{B}: \mathrm{P}=0.0017)$. $\mathrm{C}-\mathrm{F}$ : Performance in the $\mathrm{BWB}$, tethered animals showed no differences between groups for both the number of entries into the white box (C) as well as the number of stretching postures (E). In the telemetry group animals with epilepsy showed significantly fewer entries into the white box $(D: P=0.0017)$ and fewer stretching postures $(F: P=0.0115)$. $G \& H$ : EPM performance, in both the tethered $(G: F(2,27)=$ 8.706, $\mathrm{P}=0.0012$, epilepsy against both control groups $\mathrm{P}<0.01)$ and telemetry $(\mathrm{H}, \mathrm{P}=0.0049)$ group the animals with epilepsy spent significantly less time in the center area of the EPM compared to both control groups. I-J: saccharin preference test, in both the tethered (I: $\mathrm{F}(2,28)=12.86, \mathrm{P}=0.0001$, epilepsy against both control groups $\mathrm{P}<0.001)$ and telemetry $(\mathrm{J}: \mathrm{P}=$ 0.0036) group the animals with epilepsy showed lower preference for saccharin compared to both control groups. K \& L: Fecal corticosterone metabolite levels. Feces was collected at various stages during the study. In the tethered group $(\mathrm{K})$ elevated fecal corticosterone metabolite levels were found in the stimulated animals two days post-SE induction $(\mathrm{F}(2,30)=$ 4.975, $\mathrm{P}=0.0136$, epilepsy against both control groups $\mathrm{P}<0.05)$. In the telemetry group (L) elevated levels were found in the stimulated animals at four weeks $(\mathrm{P}=0.0073)$ and thirteen weeks post-SE $(\mathrm{P}=0.0188)$ induction. Error bars indicate standard error of the mean. ${ }^{*}=\mathrm{P}<$ $0.05, * *=\mathrm{P}<0.01$ and $* * *=\mathrm{P}<0.001$. Total $\mathrm{n}$ for the tethered group: naive $\mathrm{n}=11$, sham $\mathrm{n}=11$, Post-SE $n=12$, epilepsy $n=10$. Total $n$ for the telemetry group: sham $n=6$, epilepsy $n=7$. Total number of pairs in the social interaction test for the tethered group: naive $n=5$ pairs, sham $n=$ 5 pairs, epilepsy $n=4$ pairs and the telemetry group: sham $n=3$ pairs, epilepsy $n=3$ pairs. 
Figure 4, Home cage activity and heart rate. A \& C: Timeline depicting two days of recordings at four different time points. B \& D: Mean values calculated for both the combined day and night periods for each of the four time points. A \& B: In the early post-insult phase stimulated animals showed increased activity compared to baseline levels in the light phase $(\mathrm{P}=0.0093)$ (=resting phase), during the dark phase in the latency phase stimulated animals showed increased activity compared to baseline levels $(\mathrm{P}=0$ 0.0057). C \& $\mathrm{D}$ : Increased heart rate was observed in post-SE animals in the early phase compared to baseline levels during the day $(\mathrm{P}=$ 0.0341), furthermore, heart rate was significantly increased in the latency phase during both the day $(\mathrm{P}=0.0330)$ and night $(\mathrm{P}=0.0200)$ compared to the sham animals while post-SE animals showed lower heart rate during the night phase in the early post-insult phase compared to sham animals $(\mathrm{P}=0.0309)$. Total $\mathrm{n}$ for the telemetry group: sham $\mathrm{n}=6$, epilepsy $\mathrm{n}=5 . *=\mathrm{P}<0.05$ and $* *=\mathrm{P}<0.01$.

Figure 5, Correlation matrix. A heat map representing the spearman correlations between the majority of measured variables.

Figure 6, Principal component analyses. A PCA with all stimulated animals using variables measured following the monitoring period to dissect any influence of the tethered vs telemetric monitoring. A: The $\mathrm{x}$-axis shows principal component 1 (PC1) representing $24.80 \%$ of variance while the y-axis represents in the principal component 2 (PC2) 16.63\% of the variance. The tethered and telemetry group are diagonally significantly separated along both PC1 and PC2. B: the relative weight the individual variables have on PC1 and PC2. 


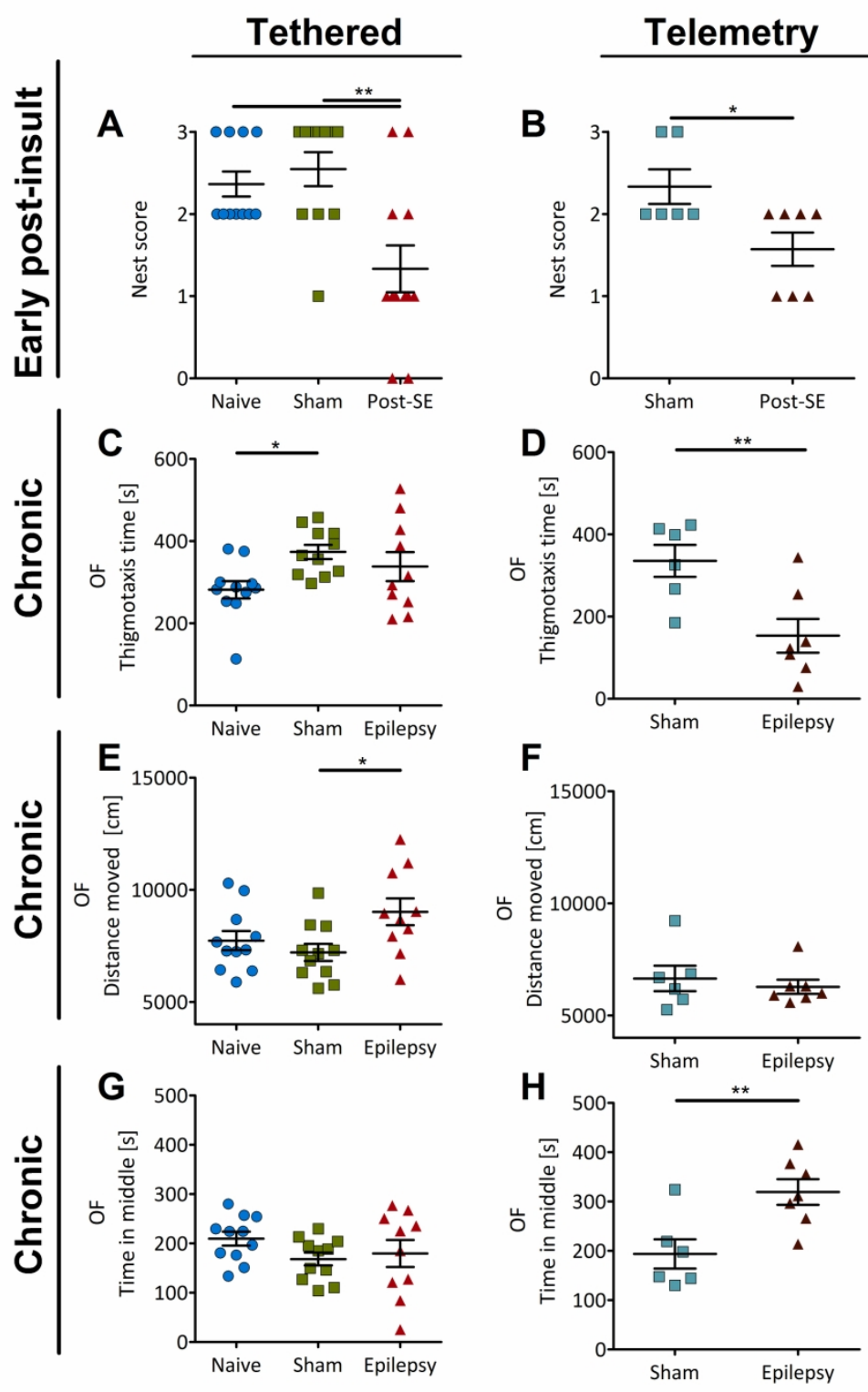

Figure 1, Nest building activity and open field locomotor activity. A \& B: Nest building scores are significantly reduced one week post-SE induction in both the tethered and the telemetry group $(A:(F 2,31)=8.591$, $\mathrm{P}=0.0048$; $\mathrm{B}: \mathrm{P}=0.0246$, epilepsy against both control groups $\mathrm{P}<0.05)$. $\mathrm{C}-\mathrm{H}$ : measures of locomotor activity measured in the open field. In the tethered group sham animals spent significantly more time at the wall compared to the naive animals $(C: F(2,29)=3.524, P=0.0427$, sham against naive $P<0.05)$ while in the telemetry group the sham animals spent significantly more time at the wall compared to the Epilepsy group $(D, P=0.0086)$. Animals with epilepsy move greater distances compared to the sham group in the tethered group $(E: F(2,29)=3.791, P=0.2073$, epilepsy against sham $P<0.05)$ but not in the telemetry group $(F)$. Lastly, animals with epilepsy spent more time in the middle of the arena compared to the sham group in the telemetry group $(\mathrm{H}: \mathrm{P}=0.0086)$ while no differences were found in the thered group $(\mathrm{G})$. Error bars indicate standard error of the mean. $*=P<0.05$ and $* *=P<0.01$. Total $n$ for the tethered group: naive $n=11$, sham $n=11$, Post-SE $n=12$, epilepsy $n=10$. Total $n$ for the telemetry group: sham $n=6$, epilepsy $n=7$. 

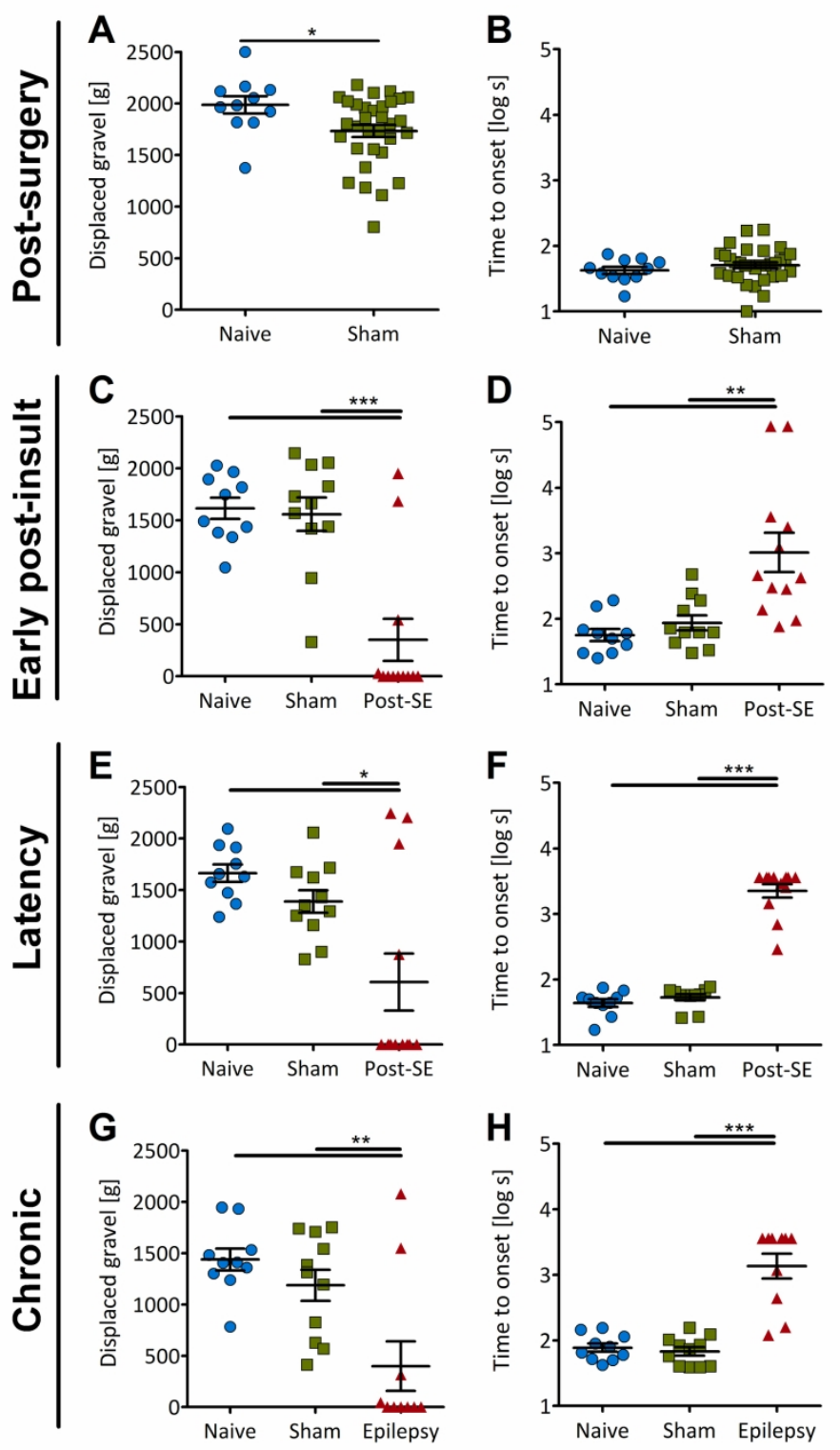

Figure 2, Burrowing behavior in animals monitored with a tethered connection. A, C, E \& G: amount of burrowed gravel, B, D, F \& H: latency to start burrowing. Animals burrowed significantly less gravel one week post-surgery $(A: P=0.0313)$ and following $S E$ induction during the early phase $(C: F(2,30)=18.93, P$ $<0.0001$, epilepsy against both control groups $\mathrm{P}<0.001)$, latency phase $(E: F(2,30)=8.438, P=0.0002$, epilepsy against both control groups $P<0.05)$ and the chronic phase $(G: F(2,28)=9.477, P=0.0007$, epilepsy against both control groups $\mathrm{P}<0.01)$ compared to both naive and sham animals. In addition, animals had a longer latency to start burrowing during the three phases post-SE induction ( $D: F(2,30)=11.19 P=0.0002$, epilepsy against both control groups $\mathrm{P}<0.01 ; \mathrm{F}: \mathrm{F}(2,30)=162.1, \mathrm{P}<0.0001$, epilepsy against both control groups $\mathrm{P}<0.001 \& \mathrm{H}: \mathrm{F}(2,28)=37.44, \mathrm{P}<0.0001$, epilepsy against both control groups $\mathrm{P}<0.001)$. Error bars indicate standard error of the mean. ${ }^{*}=\mathrm{P}<0.05, * *=\mathrm{P}<0.01$ and $* * *=\mathrm{P}<0.001$. Total $\mathrm{n}$ for the tethered group: naive $n=10$, sham $n=11$, Post-SE $n=12$, epilepsy $n=10$. 
42

43

44

45

46

47

48

49

50

51

52

53

54

55

56

57

58

59

60 

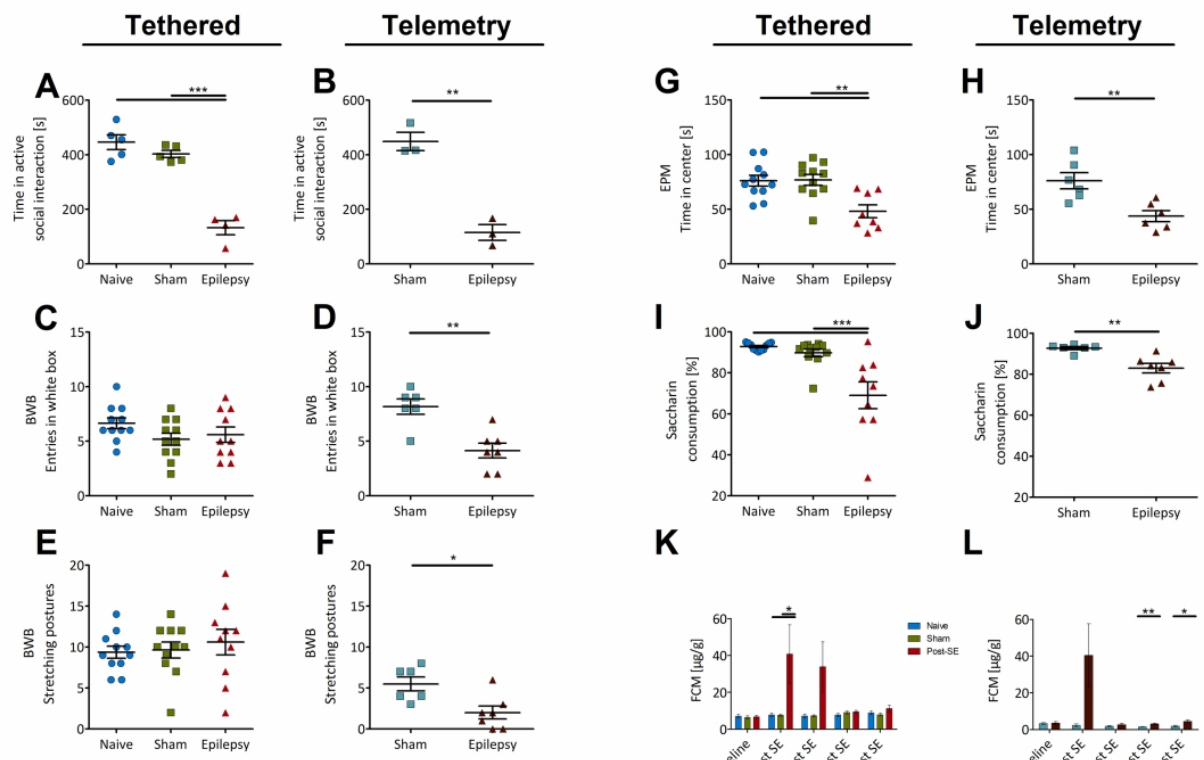

K

J
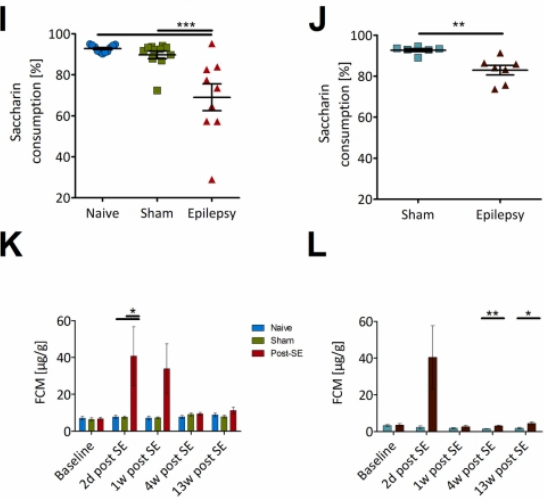

L

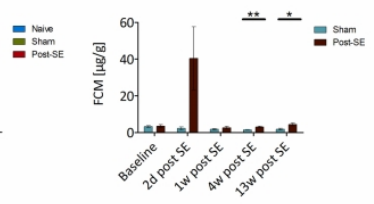

Figure 3, Social interaction, anxiety-associated, anhedonia-associated behavior and fecal corticosterone metabolite concentration (FCM). A \& B: Time spent in social interaction, animals with epilepsy spent significantly less time in active social interaction compared to control groups in both the tethered ( $A$ : $\mathrm{F}(2,11)=52.32, \mathrm{P}<0.0001$, epilepsy against both control groups $\mathrm{P}<0.001)$ as well as the telemetry group ( $B$ : $P=0.0017) . C-F$ : Performance in the $B W B$, tethered animals showed no differences between groups for both the number of entries into the white box $(C)$ as well as the number of stretching postures $(E)$. In the telemetry group animals with epilepsy showed significantly fewer entries into the white box ( $D: P=0.0017)$ and fewer stretching postures ( $F: P=0.0115)$. $G \& H$ : EPM performance, in both the tethered (G: $F(2,27)=$ 8.706, $P=0.0012$, epilepsy against both control groups $P<0.01)$ and telemetry $(H, P=0.0049)$ group the animals with epilepsy spent significantly less time in the center area of the EPM compared to both control groups. I-J: saccharin preference test, in both the tethered ( $\mathrm{I}: \mathrm{F}(2,28)=12.86, \mathrm{P}=0.0001$, epilepsy against both control groups $\mathrm{P}<0.001)$ and telemetry $(\mathrm{J}: \mathrm{P}=0.0036)$ group the animals with epilepsy showed lower preference for saccharin compared to both control groups. K \& L: Fecal corticosterone metabolite levels.

Feces was collected at various stages during the study. In the tethered group $(\mathrm{K})$ elevated fecal corticosterone metabolite levels were found in the stimulated animals two days post-SE induction $(F(2,30)=$ $4.975, P=0.0136$, epilepsy against both control groups $P<0.05)$. In the telemetry group $(L)$ elevated levels were found in the stimulated animals at four weeks $(P=0.0073)$ and thirteen weeks post-SE $(P=0.0188)$ induction. Error bars indicate standard error of the mean. $*=\mathrm{P}<0.05, * *=\mathrm{P}<0.01$ and $* * *=\mathrm{P}<$ 0.001 . Total $n$ for the tethered group: naive $n=11$, sham $n=11$, Post-SE $n=12$, epilepsy $n=10$. Total $n$ for the telemetry group: sham $n=6$, epilepsy $n=7$. Total number of pairs in the social interaction test for the tethered group: naive $n=5$ pairs, sham $n=5$ pairs, epilepsy $n=4$ pairs and the telemetry group: sham $n=3$ pairs, epilepsy $n=3$ pairs. 

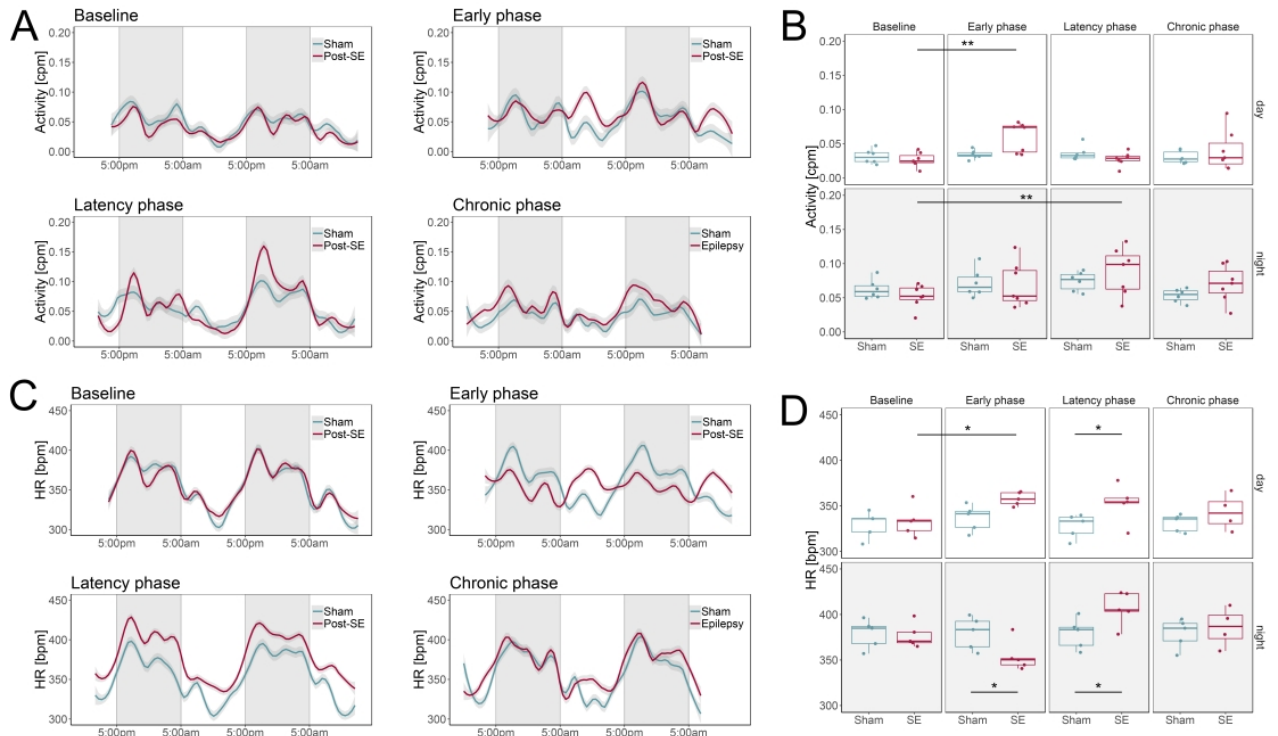

Figure 4, Home cage activity and heart rate. A \& C: Timeline depicting two days of recordings at four different time points. B \& D: Mean values calculated for both the combined day and night periods for each of the four time points. A \& B: In the early post-insult phase stimulated animals showed increased activity compared to baseline levels in the light phase $(P=0.0093)$ (=resting phase), during the dark phase in the latency phase stimulated animals showed increased activity compared to baseline levels $(P=0 \quad 0.0057)$. C \& $D$ : Increased heart rate was observed in post-SE animals in the early phase compared to baseline levels during the day $(P=0.0341)$, furthermore, heart rate was significantly increased in the latency phase during both the day $(P=0.0330)$ and night $(P=0.0200)$ compared to the sham animals while post-SE animals showed lower heart rate during the night phase in the early post-insult phase compared to sham animals $(\mathrm{P}=0.0309)$. Total $\mathrm{n}$ for the telemetry group: sham $\mathrm{n}=6$, epilepsy $\mathrm{n}=5$. $*=\mathrm{P}<0.05$ and $* *=\mathrm{P}<0.01$. 
Figure 5, Correlation matrix. A heat map representing the spearman correlations between the majority of measured variables. 


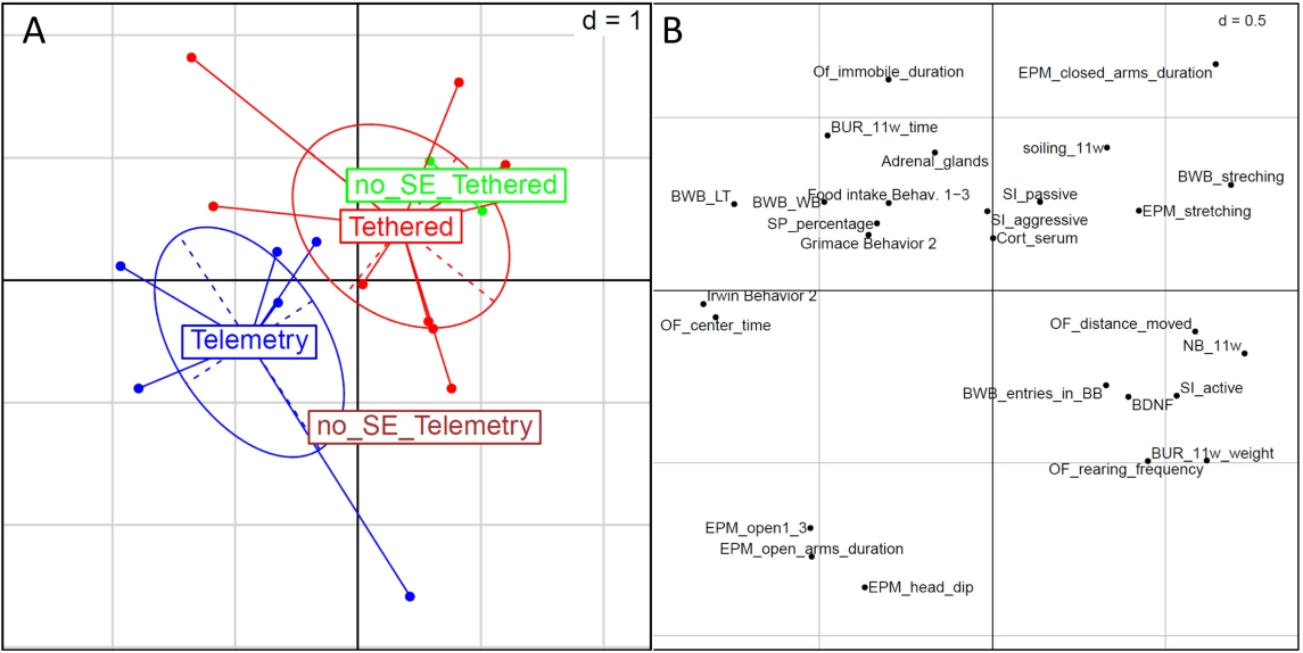

Figure 6, Principal component analyses. A PCA with all stimulated animals using variables measured following the monitoring period to dissect any influence of the tethered vs telemetric monitoring. A: The $x-$ axis shows principal component 1 (PC1) representing $24.80 \%$ of variance while the $y$-axis represents in the principal component 2 (PC2) $16.63 \%$ of the variance. The tethered and telemetry group are diagonally significantly separated along both PC1 and PC2. B: the relative weight the individual variables have on PC1 and PC2. 